\title{
Rabaska
}

Revue d'ethnologie de l'Amérique française

LABERGE, MARC. Affiquets, matachias et vermillon.

Ethnographie illustrée des Algonquiens du nord-est de

l'Amérique aux XVI ${ }^{e}, \mathrm{XVII}^{e}$ et XVIII ${ }^{e}$ siècles, Montréal,

Recherches amérindiennes au Québec, "Signes des

Amériques » 11, [2010], 227 p. Illustrations de FRANÇOIS

GIRARD. ISBN 978-2-920366-38-1

\section{Vanessa Ferey}

\section{Volume 11, 2013}

URI : https://id.erudit.org/iderudit/1018540ar

DOI : https://doi.org/10.7202/1018540ar

Aller au sommaire du numéro

\section{Éditeur(s)}

Société québécoise d'ethnologie

ISSN

1703-7433 (imprimé)

1916-7350 (numérique)

Découvrir la revue

Citer ce compte rendu

Ferey, V. (2013). Compte rendu de [LABERGE, MARC. Affiquets, matachias et vermillon. Ethnographie illustrée des Algonquiens du nord-est de l'Amérique aux $\mathrm{XVI}^{e}, \mathrm{XVII}^{e}$ et XVIII ${ }^{e}$ siècles, Montréal, Recherches amérindiennes au Québec, "Signes des Amériques " 11, [2010], 227 p. Illustrations de FRANÇOIS GIRARD. ISBN 978-2-920366-38-1]. Rabaska, 11, 217-221.

https://doi.org/10.7202/1018540ar d'utilisation que vous pouvez consulter en ligne. 
livre. Au cœur de toute cette production se trouvent les gwerzioù : un corpus étonnant de chansons qui mettent en vedette la vie d'hommes, de femmes et d'enfants, artisans et paysans, petit peuple et noblesse face à la tragédie, la perte, la violence et la mort. Comme tel, le sujet transcende les frontières de la Basse-Bretagne pour prendre une valeur universelle. L'application des outils de recherche suggère que la méthodologie mise au point par Éva Guillorel aura aussi des répercussions bien au-delà du contexte spécifique de la Basse-Bretagne et que désormais cet ouvrage de référence fera partie de la trousse de tout chercheur qui s'intéresse aux traditions orales.

Marcel Bénéteau

Université de Sudbury

LABerge, Marc. Affiquets, matachias et vermillon. Ethnographie illustrée des Algonquiens du nord-est de l'Amérique aux XVI ${ }^{e}$, XVI' et XVIII siècles, Montréal, Recherches amérindiennes au Québec, "Signes des Amériques » 11, [2010], 227 p. Illustrations de FrançOIS GIRARD. ISBN 9782-920366-38-1.

Cet ouvrage de Marc Laberge est tiré de sa thèse de doctorat en Arts et traditions populaires à l'Université Laval, réalisée sous le titre de Création d'une nouvelle iconographie sur les Algonquiens du nord-est de l'Amérique à partir des données ethnohistoriques datant d'avant 1760. Il est aujourd'hui une référence dont toute recherche en culture matérielle d'Amérique du Nord ne saurait se passer. Tant incontournable par l'originalité de la démarche que par la qualité du traitement du sujet, Affiquets, matachias et vermillon offre un regard inédit sur les communautés algonquiennes d'un passé colonial, mais aussi d'un présent engagé et d'un futur prometteur.

La préface de Jean Simard, codirecteur de thèse avec Denys Delâge, amorce un questionnement sur l'image de l'«Amérindien ancien » devenue suspecte au regard des travaux critiques de l'anthropologie historique actuelle. En effet, la démarche scientifique est en grande partie problématisée autour du fait que les films, les manuels scolaires ainsi que les romans illustrés ont largement répandu une figure stéréotypée de l'«Indien », entre agressivité barbare et naïveté naturaliste. Afin de faire fi des préjugés, quoi de plus efficace que d'établir l'iconographie des Amérindiens anciens par la justification d'un travail documentaire à la hauteur des moyens et des exigences scientifiques d'aujourd'hui ? D'emblée le préambule annonce l'origine de la démarche de l'auteur. Au courant des années 1970, Marc Laberge est photographe et vidéaste lors de fouilles archéologiques sur des sites préhistoriques et historiques. Depuis les besoins de vulgarisation des archéologues naît alors 
la conviction que cette image contemporaine est nécessaire, mais absente. Un voyage au long cours vers une nouvelle représentation visuelle des us et coutumes des Algonquins s'inscrit en perspective de ses activités artistiques et professionnelles.

Sa méthodologie est dépeinte afin de comprendre toute la complexité du projet. Les musées sont parmi les destinations privilégiées afin de constituer une solide base de diapositives d'artefacts et documents de recherche pouvant aider à la réalisation des dessins. L'American Indien Museum et l'American Museum of Natural History (New-York), la Smithsonian Institution (Washington), l'ancien Musée des civilisations du Canada (Ottawa) ou encore le Musée d'archéologie de Trois-Rivières ainsi que des collections appartenant à l'époque au Ministère de la Culture (Québec) se prêtent au jeu de la constitution d'un fonds de documentation visuelle. Néanmoins, la tâche s'avère rapidement difficile. De façon compréhensible, les musées ne renferment pas toutes les pièces nécessaires à la réalisation de l'ethnographie illustrée. Faut-il à ce stade combler les manques informationnels en ayant recours à des artifices de mises en scène et de conception d'images, voire d'effets visuels de style ? Manifestement non. Le style « impressionniste » risquerait de flouer la qualité de la recherche en bornant ses résultats à une iconographie simplement « évocatrice», sévèrement exploitée par les manuels scolaires d'histoire canadienne et rendue abusivement célèbre par les dessins de la collection Time Life.

En conséquence, les acteurs du projet de Marc Laberge s'attaquent aux problèmes de la représentation figurative de manière radicale. Le style doit être « réaliste » à partir de l'image présente dans le contexte idéologique de la société québécoise contemporaine. L'interrogation principale à ce stade de la recherche est : quelle représentation avons-nous de nos rapports avec le passé ainsi qu'avec la situation actuelle des communautés algonquiennes? Il faut donc effectuer des « choix » sur ce qui doit être créé dans l'iconographie : quels événements, gestes ou actions faire apparaître ? Le chercheur demeure ainsi en quête d'honnêteté dans sa démarche d'investigation. Il tisse en préambule un pacte de confiance avec son lectorat, conscient de ne pas prétendre à une objectivité impartiale, de ne pas échapper totalement à son époque malgré le souci du recul scientifique. Le choix de la famille linguistique algonquienne contribue également à l'objectivité puisque l'exercice semble simplifié par la sélection de cet ensemble socioculturel relativement homogène. Idem pour le choix de l'échelle de temps historique, du contact européen à 1760. L'homogénéité des fonds documentaires disponibles permet à l'auteur de bâtir son étude sur des informations plus fiables que seulement celles recueillies à partir de matériaux préhistoriques. Il faut noter que le mode 
de transmission de connaissances chez les Amérindiens n'offre pas plus de possibilités concernant la période de la préhistoire.

Ce deuil scientifique étant clairement admis, Marc Laberge s'attèle à la définition de l'image ancienne en questionnant tour à tour sa réalité puis sa théâtralité. La création iconographique est savamment étudiée afin de comprendre le processus identique à celui entrepris ici dans son origine et sa longévité. Par une revue de littérature contemporaine, le lecteur entrevoit ainsi la difficile tâche des artistes peintres des $\mathrm{XVIII}^{\mathrm{e}}$, $\mathrm{XIX}^{\mathrm{e}}$ et $\mathrm{XX}^{\mathrm{e}}$ siècles à concevoir des représentations des Amérindiens. Leurs observations quotidiennes et expériences de voyage en tant qu'explorateurs ou missionnaires forment le socle d'un point de vue scientifique évolutif dans le temps. Cette base iconographique est mise à rude épreuve lorsqu'elle est augmentée de représentations artistiques élaborées à partir de ce type de récits, sans contact direct avec la réalité des scènes et selon des schèmes généralement propres à l'Europe.

Les travaux de la décennie 1979 à 1989 de Gilles Thérien, Les Figures de l'Indien, mais aussi de Sylvie Vincent et Bernard Arcand, L'Image de l'Amérindien dans les manuels scolaires du Québec, ainsi que ceux de François-Marc Gagnon tels que Ces hommes dits "Sauvages », sont pris en références afin de comprendre la notion de « Sauvage » des historiens depuis le $\mathrm{XVII}^{\mathrm{e}}$ siècle. Les différentes formes de représentations oscillent ainsi entre des «types » identifiables : l'« Indien naturel» (presque nu et naï), le « barbare » (cruel et sale) ou encore le noble guerrier (courageux et stoïque, mais irrémédiablement dépassé par la civilisation). Ces illustrations d'hommes vêtus à la mode française ou romaine servaient et fonctionnent encore comme référence pour notre société occidentale. Elles continuent d'affirmer par leur préservation ce qui ne devait pas définir ses membres, soit des « bons sauvages », des « errants naturels quasi animaux » sinon des « brutes sanguinaires », et ce en fonction des besoins de conquête et d'établissement des Européens, d'identification et de reconnaissance des Américains. De plus, les Québécois ont eux aussi récupéré et refaçonné cette image dont le résultat est une partie intégrante de l'imaginaire actuel. Si l'iconographie ancienne est subjective, "n'en est-il pas toujours ainsi ? » Dans l'image, l'objet et le mode de représentation de l'Autre choisi par l'auteur demeure toujours révélateurs du traitement du sujet effectué par rapport au Soi alors exploré dans le dessin. La représentation « de » l'Amérindien prévaut donc sur celle « d'un » Amérindien.

Favorablement, l'avancée des techniques en iconographie collabore fortement à l'évolution de l'illustration depuis cette pensée idéologique du colonisateur trahie par la représentation théâtrale des autochtones. Les nuances 
graphiques s'accentuent au fil du temps et la qualité des traits des artistes ne diminue plus au fil de la reproduction technique des dessins. Afin d'établir un juste équilibre entre connaissances et perceptions liées à nos moyens de communications actuelles, l'auteur convoque ici l'iconographie comme moyen de communication à part entière. L'entreprise collective va donc mettre à mal la représentation " globale et entachée de doute » en misant sur la constitution sur le long terme d'une «banque de données ethnohistoriques illustrée ». La finalité de l'approche singulière choisie s'offre aux publications sur les Amérindiens et réside également dans une utilisation auprès des musées et centres d'interprétation dans leurs contenus scientifiques d'exposition. Les productions audiovisuelles y sont également invitées.

De ce fait, la thèse de Marc Laberge n'est donc pas l'œuvre d'une « démonstration », mais bien la création d'un « outil » documentaire s'inscrivant dans une dynamique constante. Le processus déclenché à l'intérieur des paramètres définis de l'iconographie actuelle relève ainsi d'une méthode expérimentale faite d'essais, mais aussi d'erreurs. Le passage des documents écrits en hypothèses graphiques débute par la transmission aux illustrateurs de multiples indications précises : " Voilà le type d'habitation à illustrer; voilà ce à quoi ressemble un séchoir à poisson... » Si l'ambition est grande, la hauteur des attentes scientifiques l'est tout autant. Il faut en effet élaborer à rebours, les images transposées par écrit des découvreurs au moment des premiers contacts avec les autochtones. Les prémisses de recherche sont orientées sur l'inventaire des témoignages des XVI ${ }^{\mathrm{e}}, \mathrm{XVII}^{\mathrm{e}}$ et $\mathrm{XVIII}^{\mathrm{e}}$ siècles. Par l'entreprise de comparaisons et de critiques appuyées, le socle d'un solide corpus se forme peu à peu via un dépouillement systématique et un rassemblement ordonné des sources datant de la Nouvelle-France.

Dans une première partie dévoilant « Des sources écrites à l'image reconstituée », l'auteur explique en quoi l'iconographie ancienne des premiers écrits participe à une image globale qui relève du mythe. Les documents écrits y sont présentés et le passage de l'écrit au visuel est décortiqué dans sa démarche complète. Les sources sont toujours très clairement apparentes afin de comprendre la redéfinition d'une image depuis l'information jusqu'à l'analyse correspondante. Le lecteur ne manque pas non plus de suivre la fabrication de l'esquisse jusqu'au dessin final. Dans une seconde ouvrant « Une nouvelle perception », les résultats de la cueillette de données ainsi que leur analyse sont généreusement offerts. Les chapitres sont agencés afin de former l'outil de référence attendu et les données y sont classées et organisées de manière logique et donc aisément accessible. La rencontre s'opère naturellement. L'aspect physique des individus est le plus illustré de la recherche afin d'amorcer clairement la redécouverte de l'Homme ou de la Femme amérindiens avant l'explication de la répartition de leurs rôles et de 
leurs activités. L'habitation puis les activités de subsistance attisent la curiosité jusqu'à l'explication en profondeur de l'alimentation. Les multiples formes de déplacements et les divers modes de transports concluent une partie si riche de sens que de formes, et le dégradé de gris des représentations explicatives ne ternit en rien la vivacité de leurs traits. Le support visuel de l'écrit ne se trouve pas seulement dans les dessins, mais dans une syntaxe fortement optique. La synthèse et la démarche accomplie apparaissent alors sous une «Une nouvelle iconographie » avec le traitement détaillé de l'information (leur fiabilité, les difficultés taxonomiques, le double sens du mot « image », etc.) ainsi que les techniques utilisées (le recours aux modèles humains, les matériaux ethnologiques, archéologiques et iconographiques). Par ailleurs, des interrogations et éléments de réflexion sont identifiables en plus des connaissances acquises. La réévaluation iconographique est objectivée par l'analyse poussée d'une illustration tirée des œuvres de Champlain. Enfin, un lexique vient parfaire la conclusion de ce qu'est l'« Élaboration d'une iconographie » qui contribue à une perception actuelle des Algonquiens.

Réimprimée à deux reprises, la dernière version de l'ouvrage comporte désormais un fichier numérique où les dessins de François Girard sont d'autant accessibles et échangeables, ranimant la dimension interactive de l'étude. La recherche menée par Marc Laberge s'adresse aux muséologies, pédagogies et cinématographies de demain qui trouveront dans cette étude une référence iconographique pensée pour faciliter de nombreux traitements documentaires. Elle est alors aussi une forme d'invitation à la réalisation de projets d'avenir avec les communautés autochtones.

VANessa Ferey

Université du Québec à Montréal — Paris 3, Sorbonne Nouvelle

Lafrance, Louise [dir.], Nicole Bourgault, Hélène Bourque, Claude CorRiveau et Ginette Laroche. Le Patrimoine religieux de l'église SaintCharles-Garnier. Québec, Fabrique Saint-Charles-Garnier, 2012, 136 p. ISBN 978-2-9813418-0-8.

Le Patrimoine religieux de l'église Saint-Charles-Garnier est une étude qui fait suite à des recherches effectuées en 2009 et 2010 ainsi qu'à l'intérêt manifesté par les publics lors d'activités de diffusion. L'ouvrage, produit sous la direction de la Fabrique, est un collectif de femmes provenant de disciplines telles que l'histoire de l'art, l'histoire de l'architecture, l'ethnologie et la muséologie. Chacun des chapitres, sous la responsabilité d'un auteur, est donc coloré selon ses intérêts et sa formation. L'ensemble comprend sept chapitres. Les deux premiers offrent une perspective historique sur la fon- 